\title{
Exact Derivation of the Radiation Law of Antennas Embedded into Generic Nonlocal Metamaterials: A Momentum-Space Approach
}

\author{
Said Mikki*, \\ *Dept. of ECECS, University of New Haven, West Haven, Connecticut, United States, said.m.mikki@gmail.com*
}

\begin{abstract}
We solve the problem of how antennas radiate into generic nonlocal metamaterials by using a momentum-space formalism to rigorously derive the general radiation formula. The energy per Hertz by unit solid angle is computed by first deriving the dyadic Green's function of nonlocal media in the momentum space. We show that due to causality only the antihermitian part of the dyad will contribute to the radiation field. We avoid any spectral integration or using the Poynting vector (the latter known to be already inadequate in nonlocal media) by working directly with momentum space formulation and derive analytically the exact expression. The final result depends only on the modal analysis of the metamaterial.
\end{abstract}

Index Terms-Future antennas, momentum space, nonlocal metamaterials.

\section{INTRODUCTION}

The main objective of this paper is to formulate the main themes of traditional antenna theory in a language conductive to research on novel and future types of antennas, in particular those operating in complex nonclassical environments best described by a nonlocal electromagnetic material response function. Nonlocality includes most prominently spatial dispersion, i.e., the dependence of the material response function on the wavevector $\mathbf{k}$ besides the classical (temporal) dispersion characterized by the appearance of another dependence on $\omega$, the circular frequency [1]-[3]. Inspired by the earliest formulation of the problem of electromagnetic wave propagation in spatially-dispersive media, we adopt the Fourier space approach to solving and studying the less-known problem of antenna analysis and design in such media. The Fourier space approach replaces the frequency domain formulation where the fields are considered in the frequency-space domain, i.e., functions in the form $\mathbf{F}(\mathbf{r}, \omega)$, by moving to a fullyfledged 4-dimensional Fourier space where all fields (electromagnetic fields and their current sources) take the form $\mathbf{F}(\mathbf{k}, \omega)$. Following the common convention in physics, we capture the dependence on $\mathbf{k}$ by the term momentum space since momentum $\mathbf{p}$ and the wavevector $\mathbf{k}$ are related to each other in quantum physics by mere constant (the de Broglie relation $\mathbf{p}=\hbar \mathbf{k})$. Some previous work on controlling the radiation emitted by sources embedding into metamaterials include [4]-[9], where most of the focus is on conventional metamaterials used to modify the emission characteristics o optical sources in metamaterials. However, all conventional metamaterials exhibit spatial dispersion so the subject has been taken into consideration in more recent works such as [10].

The momentum space formulation of electromagnetic theory is extensively used in diverse disciplines, including condensed-matter physics [11], plasma physics [3], quantum field theory [12], quantum optics [13]. However, momentum space does not seem to have been widely used in classical antenna theory where most treatments tend to favor the frequency-space formulation, with some exceptions like [14], [15]-[17]. For example, the plane-wave spectrum, a momentum representation of EM fields, was deployed for applications to near-field measurement [18], computation of Green's functions in inhomogeneous media [19], [20], subwavelength imaging [21], and characterizing mutual coupling and interactions [14], [16], [22], [23]. Periodic structures are examples of systems in which wave propagation analysis is fundamentally conducted in the spatial Fourier space, although in that case it is usually referred to as reciprocal space [24]. In this paper, we propose a momentum space formalism for antenna theory adapted from some of the original applications in physics but now for the needs of antenna theory in engineering. Our goal is to sketch out in broad manner the general ideas, basically how to define radiation patterns and array theory in momentum space instead of time-space or frequency-space. The main application of the theory is for future antennas utilizing metamaterials exhibiting nonlocal behaviour, where in that particular case we argue that the Fourier space approach provides the best means to tackle the subject [17], [25].

\section{The DyAdic GREen's FunCtion OF NONLOCAL Media in MOMEnTUM SPACE}

The 4-dimensional Fourier transform of a generic vector field $\mathbf{F}(\mathbf{r}, t)$ in space-time is defined by

$$
\mathbf{F}(\mathbf{k}, \omega):=\int_{\mathbb{R}^{4}} \mathrm{~d}^{3} r \mathrm{~d} t \mathbf{F}(\mathbf{r}, t) e^{-\mathrm{i} \mathbf{k} \cdot \mathbf{r}+\mathrm{i} \omega t} .
$$

If the field $\mathbf{F}$ is well-behaved in $\mathbb{R}^{4}$, then the inverse Fourier integral exist giving

$$
\mathbf{F}(\mathbf{r}, t)=\int_{\mathbb{R}^{4}} \frac{\mathrm{d}^{3} k \mathrm{~d} \omega}{(2 \pi)^{4}} \mathbf{F}(\mathbf{k}, \omega) e^{\mathrm{i} \mathbf{k} \cdot \mathbf{r}-\mathrm{i} \omega t} .
$$

Throughout this paper, we assume that all relevant electromagnetic fields and currents in nonlocal material domains possess 
Fourier transforms in the sense that the pair (1) and (2) exist. ${ }^{1}$ In the Fourier domain, Maxwell's equations becomes

$$
\begin{gathered}
\mathbf{k} \times \mathbf{E}(\mathbf{k}, \omega)=\omega \mathbf{B}(\mathbf{k}, \omega), \\
\mathrm{i} \mathbf{k} \times \mathbf{B}(\mathbf{k}, \omega)=\mu_{0} \mathbf{J}(\mathbf{k}, \omega)-\left(\mathrm{i} \omega / c^{2}\right) \mathbf{E}(\mathbf{k}, \omega), \\
\mathbf{k} \cdot \mathbf{E}(\mathbf{k}, \omega)=-\mathrm{i} \rho(\mathbf{k}, \omega) / \varepsilon_{0}, \mathbf{k} \cdot \mathbf{B}(\mathbf{k}, \omega)=0,
\end{gathered}
$$

where $\mathbf{E}(\mathbf{k}, \omega), \mathbf{B}(\mathbf{k}, \omega)$, and $\mathbf{J}(\mathbf{k}, \omega)$ are the space-time Fourier transforms of the electric field, magnetic flux intensity, and the source current distribution, respectively. The relations (3)-(5) are valid in arbitrary material domain with both temporal and spatial dispersion. However, they cannot be solved till we provide a description of the material response function. In this paper, we follow the Fourier transform approach in defining the constitutive relation and therefore will not use the much more familiar approach through the multipole expansion often deployed in the engineering literature. In particular, we decompose the total current $\mathbf{J}(\mathbf{k}, \omega)$ into two parts

$$
\mathbf{J}(\mathbf{k}, \omega)=\mathbf{J}_{\text {ant }}(\mathbf{k}, \omega)+\mathbf{J}_{\text {ind }}(\mathbf{k}, \omega),
$$

where $\mathbf{J}_{\text {ant }}(\mathbf{k}, \omega)$ is the externally supplied antenna current distribution while $\mathbf{J}_{\text {ind }}(\mathbf{k}, \omega)$ is the current induced in the medium as a response to the excitation electric field, i.e., we write

$$
\mathbf{J}_{\text {ind }}(\mathbf{k}, \omega)=\overline{\bar{\sigma}}(\mathbf{k}, \omega) \cdot \mathbf{E}(\mathbf{k}, \omega),
$$

where $\overline{\bar{\sigma}}(\mathbf{k}, \omega)$ is the material conductivity tensor [11]. Here, spatial dispersion (nonlocality) is captured by the dependence of the conductivity on $\mathbf{k}$, while the appearance of $\omega$ reflects normal or temporal dispersion [1], [2]. The relation between the electric displacement vector $\mathbf{D}(\mathbf{k}, \omega)$ and the electric field can now be expressed by

$$
\mathbf{D}(\mathbf{k}, \omega)=\overline{\bar{\varepsilon}}(\mathbf{k}, \omega) \cdot \mathbf{E}(\mathbf{k}, \omega), \quad \overline{\bar{\varepsilon}}(\mathbf{k}, \omega)=\overline{\mathbf{I}}+\frac{\mathrm{i}}{\omega \varepsilon_{0}} \overline{\bar{\sigma}}(\mathbf{k}, \omega) .
$$

Here, $\overline{\mathbf{I}}$ is the unit dyad. The tensor $\overline{\bar{\varepsilon}}(\mathbf{k}, \omega)$ in (8) is called the equivalent dielectric function of the medium in frequency-momentum space [2], [25]. It provides the most general description of the nonlocal medium in the frequencymomentum space [11]. ${ }^{2}$ It enjoys several properties that are based on universal principles such energy conservation, causality, reciprocity, etc, hence valid irrespective to the actual microscopic details of the medium [2], [11], [17]. We mention here only those related to dissipation and non-dissipation because they will pop out frequently in the antenna theory to be developed here. The material response tensor in general can be expanded as $\overline{\bar{\varepsilon}}(\mathbf{k}, \omega)=\overline{\bar{\varepsilon}}^{\mathrm{H}}(\mathbf{k}, \omega)+\overline{\bar{\varepsilon}}^{\mathrm{A}}(\mathbf{k}, \omega)$, where here we define $\overline{\bar{\varepsilon}}^{\mathrm{H}}(\mathbf{k}, \omega):=(1 / 2)\left[\overline{\bar{\varepsilon}}(\mathbf{k}, \omega)+\overline{\bar{\varepsilon}}^{*}(\mathbf{k}, \omega)\right]$ and $\overline{\bar{\varepsilon}}^{\mathrm{A}}(\mathbf{k}, \omega):=(1 / 2)\left[\overline{\bar{\varepsilon}}(\mathbf{k}, \omega)-\overline{\bar{\varepsilon}}^{*}(\mathbf{k}, \omega)\right]$, i.e., the hermitian and antihermitian parts, respectively. In component form, it is clear that $\overline{\bar{\varepsilon}}_{n m}^{\mathrm{H}}=\overline{\bar{\varepsilon}}_{m n}^{\mathrm{H} *}$ and $\overline{\bar{\varepsilon}}_{n m}^{\mathrm{A}}=-\overline{\bar{\varepsilon}}_{m n}^{\mathrm{A} *}$. It can be shown that only the antihermitian part of the response functions $\overline{\bar{\sigma}}$

\footnotetext{
${ }^{1}$ For further details about the precise mathematical conditions, see [12].

${ }^{2}$ In contrast to the traditional multipole approach, the Fourier space approach to the electromagnetic response of material domains include all electric and magnetic responses in one response tensor, namely the tensor $\overline{\bar{\varepsilon}}$.
}

and $\overline{\bar{\varepsilon}}$ actually contribute to dissipative processes such as wave growth or decay inside the medium [2], [11], [13].

The fundamental assumption of the momentum space antenna theory is that the antenna current is an independent function externally imposed from the "outside" of the material, i.e., in contrast to $\mathbf{J}_{\text {ind }}$, the antenna current $\mathbf{J}_{\text {ant }}$ is not determined by microscopic processes immanent to the nonlocal material system itself. On the other hand, the induced current $\mathbf{J}_{\text {ind }}$ collects all individual processes in the material system produced in response to the applied external source, e.g., polarization current, conductive current, magnetization, etc. To find the electromagnetic fields produced by the antenna current source $\mathbf{J}_{\text {ant }}$, the vector magnetic potential $\mathbf{A}(\mathbf{r}, t)$ and the scalar electric potential $\phi(\mathbf{r}, t)$ are often introduced where

$$
\mathbf{E}(\mathbf{r}, t)=-\nabla \phi(\mathbf{r}, t)-\frac{\partial \mathbf{A}(\mathbf{r}, t)}{\partial t}, \mathbf{B}(\mathbf{r}, t)=\nabla \times \mathbf{A}(\mathbf{r}, t) .
$$

There is then the well-known freedom of choosing a suitable gauge condition (relation between A and $\phi$ ) since Maxwell's equations in themselves are compatible with an infinite number of valid choices of these potential functions (gauge freedom.) It turns out that for the development of antenna theory in nonlocal material domains, the best gauge condition to utilize is the Fourier gauge

$$
\text { The Fourier Gauge : } \quad \phi(\mathbf{r}, t)=0 .
$$

Consequently, in this case we have

$$
\mathbf{E}(\mathbf{r}, t)=-\frac{\partial \mathbf{A}(\mathbf{r}, t)}{\partial t}, \quad \mathbf{E}(\mathbf{k}, \omega)=\mathrm{i} \omega \mathbf{A}(\mathbf{k}, \omega)
$$

The gauge condition (10) will be used throughout this work. In particular, let us write the wave equation in the Fourier space. From (3)-(5), we easily deduce

$$
\frac{\omega^{2}}{c^{2}} \mathbf{E}(\mathbf{k}, \omega)+\mathbf{k} \times[\mathbf{k} \times \mathbf{E}(\mathbf{k}, \omega)]=-\mathrm{i} \omega \mu_{0} \mathbf{J}(\mathbf{k}, \omega) .
$$

Using (11), this leads to

$$
\frac{\omega^{2}}{c^{2}} \mathbf{A}(\mathbf{k}, \omega)+\mathbf{k} \times[\mathbf{k} \times \mathbf{A}(\mathbf{k}, \omega)]=-\mu_{0} \mathbf{J}(\mathbf{k}, \omega)
$$

Note that the remaining field and source components, namely B and $\rho$, can be determined from other equations like (3) and the equation of continuity, giving rise to

$$
\mathbf{B}(\mathbf{k}, \omega)=\frac{1}{\omega} \mathbf{k} \times \mathbf{E}(\mathbf{k}, \omega), \quad \rho(\mathbf{k}, \omega)=\frac{\mathbf{1}}{\omega} \mathbf{k} \cdot \mathbf{E}(\mathbf{k}, \omega) .
$$

In other words, in the Fourier domain, the only effective unknown is the frequency-momentum space electric field $\mathbf{E}(\mathbf{k}, \omega)$ while all other quantities can be determined based on this field variable. Moreover, using the Fourier gauge, only the momentum space vector potential $A(\mathbf{k}, \omega)$ must be found, essentially by solving (13). Equally important is that both (12) and (13) are in fact algebraic equations, allowing us to derive exact analytical expressions for the antenna radiation in nonlocal media as will be demonstrated later. ${ }^{3}$

\footnotetext{
${ }^{3}$ In the Fourier gauge, the only restriction is that $\omega \neq 0$, an assumption made here. However, for applications to antennas, this is already the case since radiation does not occur in the static regime $\omega=0$.
} 
Using (6), (7), and (11), we can introduce a slightly different material tensor $\overline{\bar{\zeta}}(\mathbf{k}, \omega)$ defined by

$$
\mathbf{J}_{\text {ind }}(\mathbf{k}, \omega)=\overline{\bar{\zeta}}(\mathbf{k}, \omega) \cdot \mathbf{A}(\mathbf{k}, \omega), \quad \overline{\bar{\zeta}}(\mathbf{k}, \omega):=\mathrm{i} \omega \overline{\bar{\sigma}}(\mathbf{k}, \omega)
$$

In terms of the tensor, the effective dielectric tensor can be written as

$$
\overline{\bar{\varepsilon}}(\mathbf{k}, \omega)=\overline{\mathbf{I}}+\frac{1}{\omega^{2} \varepsilon_{0}} \overline{\bar{\zeta}}(\mathbf{k}, \omega) .
$$

With the help of (15) and (16), the wave equation (13) can then be reexpressed in the following more compact operator form

$$
\overline{\mathbf{G}}^{-1}(\mathbf{k}, \omega) \cdot \mathbf{A}(\mathbf{k}, \omega)=-\frac{\mu_{0} c^{2}}{\omega^{2}} \mathbf{J}_{\text {ant }}(\mathbf{k}, \omega),
$$

where the dyadic tensor

$$
\overline{\mathbf{G}}^{-1}(\mathbf{k}, \omega):=-\frac{k^{2} c^{2}}{\omega^{2}}(\overline{\mathbf{I}}-\hat{k} \hat{k})+\overline{\bar{\varepsilon}}(\mathbf{k}, \omega)
$$

is the inverse of the momentum-space radiation operator (dyadic Green's function) $\overline{\mathbf{G}}(\mathbf{k}, \omega)$. Here, $k:=|\mathbf{k}|$ is the magnitude of the wavevector $\mathbf{k}$ and $\hat{k}:=\mathbf{k} / k$ is the unit vector pointing in the direction of $\mathbf{k}$. When there is no source $\left(\mathbf{J}_{\text {ant }}=0\right)$, the relation (17) reduces to

$$
\overline{\mathbf{G}}^{-1, \mathrm{H}}(\mathbf{k}, \omega) \cdot \mathbf{A}(\mathbf{k}, \omega)=0
$$

where $\overline{\mathbf{G}}^{-1, \mathrm{H}}(\mathbf{k}, \omega)$ is the hermitian part of the tensor $\overline{\mathbf{G}}^{-1}(\mathbf{k}, \omega)$. Here, we adopted the general approach in plasma and condensed-matter physics where losses (introduced in our case by dissipation in $\overline{\mathbf{G}}^{-1}(\mathbf{k}, \omega)$, ultimately caused by the antihermitian part of $\overline{\bar{\varepsilon}}(\mathbf{k}, \omega)$ via (18)) is treated as small perturbation added to the main component of the Green's function tensor, which is hermitian [1], [2], [26]. For that reason, only the hermitian part is relevant to the determination of the radiation modes. ${ }^{4}$ The latter are found by solving the equation

$$
G^{-1, \mathrm{H}}(\mathbf{k}, \omega)=0, \quad G^{-1}:=\operatorname{det}\left[\overline{\mathbf{G}}^{-1}(\mathbf{k}, \omega)\right],
$$

where 'det' is the determinant operator. In general, there exists multiple solutions to (20), each labeled by $l$ and is captured by the $l$ th mode dispersion relation put either in the form $\omega=$ $\omega_{l}(\mathbf{k})$ or $\mathbf{k}=\mathbf{k}_{l}(\omega)$. As will be illustrated next, it turns out that the antenna radiation pattern (evaluated here in the momentum space), is completely determined by the propagating modes arising from the solution of the dispersion relation (20). For that reason, the art and science of designing antennas with desired far-field radiation patterns in nonlocal metamaterials requires engineering these radiation modes.

\section{The Antenna Radiation Pattern in Momentum SPACE}

In mainstream antenna theory and the treatment of other emission processes, the conventional approach to estimating far-field radiation consists of solving Maxwell's equations (often in vacuum) to find the electric and magnetic fields

\footnotetext{
${ }^{4} \mathrm{We}$ will however drop the superscript $\mathrm{H}$ in the future whenever that does not cause confusion in order to simplify the notation.
}

in spacetime, forming the Poynting vector, then computing the radiated power by integrating the latter in space and time [27]. This approach, however, is extremely difficult to apply in generic anisotropic media, and in the case when the material tensor is also nonlocal, it is probably not possible at all to work exclusively in spacetime. In what follows, we propose an alternative short route toward building some essential components of a viable theory of antennas radiating in nonlocal domains. The key idea is that in the momentum space of Fourier transformed fields, it is much easier to work with spectral components since they acquire their purely tensor-algebraic form developed above; simultaneously, using Parseval (power) theorems, one can relate the physical meaning of some (squared) quantities in one domain to the other.

We start by noting that the nonlocal medium Green's function (18) can be expressed analytically by

$$
G_{i j}(\mathbf{k}, \omega)=\frac{C_{i j}^{\mathrm{H}}(\mathbf{k}, \omega)}{G^{-1, \mathrm{H}}(\mathbf{k}, \omega)}
$$

where $C_{i j}$ are the cofactors of the matrix representation of the tensor $\overline{\mathbf{G}}^{-1}$ satisfying $\overline{\mathbf{G}} \cdot \overline{\mathbf{C}}=\overline{\mathbf{I}} G^{-1}$. The detailed expressions are lengthy and will not be given here but can be found in good books on matrix theory. What is important for us here is that, excepting the general functional dependence of $\overline{\bar{\varepsilon}}(\mathbf{k}, \omega)$, the nonlocal medium Green's function becomes essentially a polynomial rational function in both $\mathbf{k}$ and $\omega$. The tensor $\overline{\mathbf{G}}$ is often called in the physics literature the photon propagator. Here, we just refer to it as the nonlocal medium dyadic Green's function. Even though only the hermitian part is taken into the medium Green's function (21), there still exists an antihermitian component in this Green's function that must be added in order to enforce causality [3], [26]. To see this, note that the Green's function (21) possesses poles at the solutions of the dispersion equation (20), which implies that $G_{i j}(\mathbf{k}, \omega)$ is singular. In order to find the spatio-temporal fields, one needs to invert the Fourier transform by computing (2). This then will lead to divergent integrals unless a small perturbation in the pole location is introduced, which is usually attained by replacing $\omega$ by $\omega+\mathrm{i} \epsilon$, where $\epsilon$ is a very small positive real number. More often, we write $\omega+\mathrm{i} 0$, a notation adopted hereafter. It is clear then that around the $l$ th mode, the determinant $G^{-1}$ appearing in the denominator of (21) may be approximated by

$$
G^{-1}(\mathbf{k}, \omega) \approx \frac{\partial G^{-1}(\mathbf{k}, \omega)}{\partial \omega}\left[\omega-\omega_{l}+\mathrm{i} 0\right] .
$$

Using the Plemelj formula

$$
\frac{1}{\omega+\mathrm{i} 0}=\mathcal{P} \frac{1}{\omega+\mathrm{i} 0}-\mathrm{i} \pi \delta(\omega),
$$

where $\mathcal{P}$ is the principal Cauchy value operator, the relations (21) and (22) when summed over all radiation modes jointly imply the existence of the following antihermitian component

$$
G_{i j}^{\mathrm{A}}(\mathbf{k}, \omega)=-\mathrm{i} \pi \varepsilon_{0} \sum_{l} \omega_{l} R_{l}^{i j}(\mathbf{k}) \delta\left(\omega-\omega_{l}(\mathbf{k})\right),
$$


where

$$
R_{l}^{i j}(\mathbf{k}):=\left.\frac{C_{i j}^{\mathrm{H}}(\mathbf{k}, \omega)}{\varepsilon_{0} \omega_{l}(\mathbf{k}) \partial G^{-1}(\mathbf{k}, \omega) / \partial \omega}\right|_{\omega=\omega_{l}(\mathbf{k})}
$$

is what we term the momentum-space radiation mode Green's function. It captures the $l$ th mode contribution to the $i j$ th component $(i, j=1,2,3)$ of the nonlocal medium Green's function tensor $\overline{\mathbf{G}}(\mathbf{k}, \omega)$. It turns out that only the antihemitian part of this medium Green's function as determined by (24) actually contributes to the real radiated power of any antenna. On the other hand, the hermitian part of $\overline{\mathbf{G}}(\mathbf{k}, \omega)$ contributes only to the antenna near field.

We next explicitly compute this radiation power pattern in momentum space. Unfortunately, the common method applied in antennas radiating in free space or in nondispersive media depends on the Poynting theorem interpreted as energy conservation relation. It is well known that this direct view cannot be extended without further assumptions to generally temporally dispersive media [28]. Worse still, in nonlocal (spatially dispersive) domains, the Poynting theorem itself is not valid since power will flow along new directions emerging from higher-order corrections [2], [29], [30]. Instead, we adopt here an alternative method due to Brillouin [31] and often adopted in various settings [32]. The key idea is to estimate the energy transfered from the source to the near field right at the source and equate this with the net (real) power delivered to the medium. To achieve this in momentum space, we introduce a new radiation pattern intensity $U_{l}(\mathbf{k})$, which is formally defined as the energy transferred from the antenna current into the lth radiation mode field in the momentum-space volume $\mathrm{d}^{3} \mathbf{k} /(2 \pi)^{3}$. Clearly, the units of this quantity will be $\mathbf{J} \cdot \mathrm{m}^{3}$. Let the antenna current source $\mathbf{J}_{\text {ant }}(\mathbf{r}, t)$ be examined within a standard time interval $[-T / 2, T / 2]$. Since radiation modes do not exchange energy with each other, we can some over all radiation intensity functions $U_{l}(\mathbf{k})$ defined above to obtain

$-\int_{-T / 2}^{T / 2} \mathrm{~d} t \int_{V_{\text {an } t}} \mathrm{~d}^{3} r \mathbf{J}_{\text {ant }}(\mathbf{r}, t) \cdot \mathbf{E}(\mathbf{r}, t)=\sum_{l} \int_{\mathbb{R}^{3}} \frac{\mathrm{d}^{3} k}{(2 \pi)^{3}} U_{l}(\mathbf{k})$,

which is more general statement of energy conservation since it does not require using the Poynting vector, the latter being insufficient when nonlocality is present. Using the Parseval (power) theorem, the relation (26) can be reexpressed in frequency-momentum space as follows:

$$
\begin{array}{r}
\mathcal{E}_{\text {rad }}:=-\int_{-T / 2}^{T / 2} \mathrm{~d} t \int_{V_{\text {ant }}} \mathrm{d}^{3} r \mathbf{J}_{\text {ant }}(\mathbf{r}, t) \cdot \mathbf{E}(\mathbf{r}, t) \\
=\int_{\mathbb{R}^{4}} \frac{\mathrm{d} \omega \mathrm{d}^{3} k}{(2 \pi)^{4}} \mathbf{J}_{\text {ant }}(\mathbf{k}, \omega) \cdot \mathbf{E}(\mathbf{k}, \omega) .
\end{array}
$$

Using (11) and (17), (27) becomes

$$
\begin{aligned}
& -\int_{-T / 2}^{T / 2} \mathrm{~d} t \int_{V_{\text {ant }}} \mathrm{d}^{3} r \mathbf{J}_{\text {ant }}(\mathbf{r}, t) \cdot \mathbf{E}(\mathbf{r}, t) \\
& \quad=\int_{\mathbb{R}^{4}} \frac{\mathrm{d} \omega \mathrm{d}^{3} k}{(2 \pi)^{4}} \frac{\mathrm{i} \mu_{0} c^{2}}{\omega} \mathbf{J}_{\text {ant }}^{*}(\mathbf{k}, \omega) \cdot \overline{\mathbf{G}}(\mathbf{k}, \omega) \cdot \mathbf{J}_{\text {ant }}(\mathbf{k}, \omega) .
\end{aligned}
$$

The integral in the THS of (28) is real (because energy in the LHS is real), so it can be written as half its sum with the complex conjugate, which implies that only the antihermitian part of $\overline{\mathbf{G}}(\mathbf{k}, \omega)$ will contribute to the total integral. Inserting (24) then into (28), evaluating the trivial $\omega$-integral involving the delta function, and noticing that negative frequencies have identical contribution to positive frequencies, the following result is obtained:

$\mathcal{E}_{\mathrm{rad}}=\int_{\mathbb{R}^{3}} \frac{\mathrm{d}^{3} k}{(2 \pi)^{3}} \sum_{l} \mathbf{J}_{\mathrm{ant}}^{*}\left[\mathbf{k}, \omega_{l}(\mathbf{k})\right] \cdot \overline{\mathbf{R}}_{l}(\mathbf{k}) \cdot \mathbf{J}_{\mathrm{ant}}\left[\mathbf{k}, \omega_{l}(\mathbf{k})\right]$,

where the dyadic function $\overline{\mathbf{R}}_{l}(\mathbf{k})$ is defined as the 3dimensional dyad with cartesian components given by $R_{l}^{i j}(\mathbf{k})$ as per (25). Finally, by comparing (29) with (29), the following expression for the $l$ th mode radiation intensity is derived:

$$
U_{l}(\mathbf{k})=\mathbf{J}_{\text {ant }}^{*}\left[\mathbf{k}, \omega_{l}(\mathbf{k})\right] \cdot \overline{\mathbf{R}}_{l}(\mathbf{k}) \cdot \mathbf{J}\left[\mathbf{k}, \omega_{l}(\mathbf{k})\right] .
$$

The relation (30) is the main result of this paper. It expresses the amount of radiated energy within a unit volume in the momentum-space in terms of the radiation mode spectral Green's function $\overline{\mathbf{R}}_{l}(\mathbf{k})$. Next, we show some applications of this result in antenna theory.

\section{Applications: EngineEring Antenna Radiation BY NONLOCAl METAMATERials}

Nonlocal metamaterials (NL-MTMs) are defined as engineered materials exhibiting controlled nonlocal behaviour serving a preassigned function [30], [33]. Examples of NLMTMs include engineered plasma domains and arrays of nanotubes [34]-[38]. Here, we demonstrate how the previous momentum-space theory can be used to help engineer the radiation pattern of antennas embedded into such nonlocal metamaterials.

First, we convert the radiation formula (30) into a more convenient form for antenna applications. The direction of wave propagation is $\hat{k}:=\mathbf{k} / k$, so we may describe this direction by a solid angle $\Omega$. The magnitude $k=|\mathbf{k}|$ is related to frequency through the mode dispersion relation $\omega=\omega_{l}(k, \hat{k})$. It is better, however, to express the dispersion relation in the form

$$
\frac{k^{2} c^{2}}{\omega^{2}}=n_{l}^{2}(\omega, \hat{k})
$$

which is very frequently used in optics [2]. Here, $n_{l}$ is the index of refraction of the $l$ th mode and the positive square root of (31) is assumed. The volume element $\mathrm{d}^{3} k /(2 \pi)^{3}$ in momentum space can now be reexpressed in spherical coordinates, then we transform $k$ to $\omega$ using (31). Therefore,

$\int_{\mathbb{R}^{3}} \frac{\mathrm{d}^{3} k}{(2 \pi)^{3}}=\int_{0}^{\infty} \mathrm{d} \omega \int_{4 \pi} \mathrm{d} \hat{k} \frac{\omega^{2} n_{l}^{2}(\omega, \hat{k})}{(2 \pi c)^{3}} \frac{\partial}{\partial \omega}\left[\omega n_{l}(\omega, \hat{k})\right]$.

We now introduced the antenna radiation pattern $U_{l}(\omega, \hat{k})$, which is defined by

$$
\int_{\mathbb{R}^{3}} \frac{\mathrm{d}^{3} k}{(2 \pi)^{3}} U_{l}(\mathbf{k})=\int_{0}^{\infty} \mathrm{d} \omega \int_{4 \pi} \mathrm{d} \hat{k} U_{l}(k, \hat{k}) .
$$


Physically, $U_{l}(\omega, \hat{k})$ is the energy radiated in standard time interval with duration $T$ per unit frequency per unit solid angle. Using (32) and (30), we finally arrive at

$$
\begin{array}{r}
U_{l}(\omega, \hat{k})=\frac{\omega^{2} n_{l}^{2}(\omega, \hat{k})}{(2 \pi c)^{3}} \frac{\partial}{\partial \omega}\left[\omega n_{l}(\omega, \hat{k})\right] \\
\times U_{l}\left[(\omega / c) n_{l}(\omega, \hat{k}) \hat{k}\right],
\end{array}
$$

where

$$
\begin{aligned}
& U_{l}\left[(\omega / c) n_{l}(\omega, \hat{k}) \hat{k}\right] \\
& \quad=\left.\mathbf{J}_{\text {ant }}^{*}(\mathbf{k}, \omega) \cdot \overline{\mathbf{R}}_{l}(\mathbf{k}) \cdot \mathbf{J}_{\text {ant }}(\mathbf{k}, \omega)\right|_{\mathbf{k}=(\omega / c) n_{l}(\omega, \hat{k}) \hat{k}}
\end{aligned}
$$

In writing (34) and (35), we have used $\mathbf{k}=k \hat{k}$ then reexpressed $k$ in terms of $\omega$ and $\hat{k}$ with the help of (31). Consequently, the radiation mode antenna pattern intensity as function of direction and frequency is completely determined by the dispersion relation (31). Numerical computations based on this expression for some basic nonlocal materials will be reported in the full version of this paper.

\section{CONCLUSION}

We have provided a complete and rigorous derivation of an equivalent quantity that gives the amount of energy radiated by an antenna embedded into a generic nonlocal metamaterial per unit Hertz per unit solid angle. The method is based on carrying out all calculations in frequency-momentum space instead of the conventional approach in spacetime. Since the Poynting vector in nonlocal media fails to describe the direction of power flow, we computed the energy injected directly from the antenna current into the near field in order to estimate the radiation energy intensity per unit frequency per unit solid angle. It was found that the total radiation pattern is the sum of radiation functions each controlled by the corresponding longitudinal and/or transverse mode that the antenna launch into the nonlocal metamaterial. The derived expression can be completely evaluated analytically if the dispersion relation and hence the modes of the nonlocal medium are known.

\section{REFERENCES}

[1] V. L. Ginzburg, The propagation of electromagnetic waves in plasmas. Oxford,New York: Pergamon Press, 1970.

[2] V. Agranovich and V. Ginzburg, Crystal Optics with Spatial Dispersion, and Excitons. Berlin, Heidelberg: Springer Berlin HeidelbergImprint Springer, 1984.

[3] V. L. Ginzburg, Theoretical physics and astrophysics. Oxford New York: Pergamon Press, 1979.

[4] S. Enoch, G. Tayeb, P. Sabouroux, N. Guérin, and P. Vincent, "A metamaterial for directive emission," Phys. Rev. Lett., vol. 89, p. 213902 , Nov 2002.

[5] Y. Yuan, L. Shen, L. Ran, T. Jiang, J. Huangfu, and J. A. Kong, "Directive emission based on anisotropic metamaterials," Phys. Rev. A vol. 77, p. 053821, May 2008.

[6] Z.-G. Dong, H. Liu, T. Li, Z.-H. Zhu, S.-M. Wang, J.-X. Cao, S.-N. Zhu, and X. Zhang, "Modeling the directed transmission and reflection enhancements of the lasing surface plasmon amplification by stimulated emission of radiation in active metamaterials," Phys. Rev. B, vol. 80, p. 235116, Dec 2009.

[7] K. Halterman, S. Feng, and V. C. Nguyen, "Controlled leaky wave radiation from anisotropic epsilon near zero metamaterials," Phys. Rev. $B$, vol. 84, p. 075162, Aug 2011.
[8] W. J. M. Kort-Kamp, F. S. S. Rosa, F. A. Pinheiro, and C. Farina, "Spontaneous emission in the presence of a spherical plasmonic metamaterial," Phys. Rev. A, vol. 87, p. 023837, Feb 2013.

[9] K. M. Schulz, H. Vu, S. Schwaiger, A. Rottler, T. Korn, D. Sonnenberg, T. Kipp, and S. Mendach, "Controlling the spontaneous emission rate of quantum wells in rolled-up hyperbolic metamaterials," Phys. Rev. Lett., vol. 117, p. 085503, Aug 2016.

[10] M. Nyman, V. Kivijärvi, A. Shevchenko, and M. Kaivola, "Generation of light in spatially dispersive materials," Phys. Rev. A, vol. 95, p. 043802, Apr 2017.

[11] Ilinskii and L. Keldysh, Electromagnetic response of material media. New York: Springer Science+Business Media, 1994.

[12] E. Zeidler, Quantum field theory II: Quantum Electrodynamics. Berlin: Springer, 2006.

[13] O. Keller, Quantum Theory of Near-Field Electrodynamics. Berlin New York: Springer, 2011.

[14] D. Kerns, "Reviews and Abstracts - Plane Wave Scattering-Matrix Theory of Antennas and Antenna-Antenna Interactions," IEEE Antennas and Propagation Society Newsletter, vol. 21, no. 1, pp. 11-11, February 1979.

[15] S. Mikki and Y. Antar, "A theory of antenna electromagnetic near fieldpart II," IEEE Transactions on Antennas and Propagation, no. 12, pp. 4706-4724, December.

[16] - "A new technique for the analysis of energy coupling and exchange in general antenna systems," IEEE Transactions on Antennas and Propagation, vol. 63, no. 12, pp. 5536-5547, December 2015.

[17] —, New Foundations for Applied Electromagnetics: The Spatial Structure of Fields. London: Artech House, 2016.

[18] T. Hansen, Plane-Wave Theory of Time-Domain Fields: Near-Field Scanning applications. New York: IEEE Press, 1999.

[19] L. Felsen, Radiation and Scattering of Waves. Piscataway, NJ: IEEE Press, 1994.

[20] W. C. Chew, Waves and Fields in Inhomogenous Media. Wiley-IEEE, 1999.

[21] L. Novotny, Principles of Nano-Optics. Cambridge: Cambridge University Press, 2012.

[22] S. Mikki and Y. Antar, “The antenna current Green's function formalism-Part I," IEEE Trans. Antennas Propagat, vol. 9, pp. 44934504, September 2013.

[23] - "The antenna current Green's function formalism-Part II," IEEE Trans. Antennas Propagat, vol. 9, pp. 4505-4519, September 2013.

[24] L. Brillouin, Wave propagation in periodic structures, electric filters and crystal lattices. New York: Dover Publications, 1953.

[25] S. Mikki and A. Kishk, "Nonlocal electromagnetic media: A paradigm for material engineering," in Passive Microwave Components and Antennas. InTech, April 2010.

[26] L. D. Landau, Electrodynamics of continuous media. Oxford England: Butterworth-Heinemann, 1984

[27] S. A. Schelkunoff and H. T. Friss, Antennas: Theory and practice. New York; Chapman \& Hall: London, 1952.

[28] J. Schwinger et al., Classical electrodynamics. Reading, Mass: Perseus Books, 1998.

[29] S. Mikki and A. Kishk, "Electromagnetic wave propagation in dispersive negative group velocity media," in 2008 IEEE MTT-S International Microwave Symposium Digest, June 2008, pp. 205-208.

[30] S. Mikki and A. Kishk, "Electromagnetic wave propagation in nonlocal media: Negative group velocity and beyond," Progress In Electromagnetics Research B, vol. 14, pp. 149-174, 2009.

[31] L. Brillouin, “Origin of radiation resistance," Radioelectricite 3, pp. 147$152,1922$.

[32] C. Papas, Theory of electromagnetic wave propagation. New York: Dover Publications, 1988.

[33] S. Mikki and Y. Antar, "On electromagnetic radiation in nonlocal environments: Steps toward a theory of near field engineering," in 2015 9th European Conference on Antennas and Propagation (EuCAP), April 2015, pp. 1-5.

[34] S. Mikki and A. Kishk, "Theory of optical scattering by carbon nanotubes," Microwave and Optical Technology Letters, vol. 49, no. 10, pp. 2360-2364, Jul. 2007.

[35] - "Electromagnetic scattering by multi-wall carbon nanotubes," Progress In Electromagnetics Research B, vol. 17, pp. 49-67, 2009.

[36] S. Mikki and A. Kishk, "Effective medium theory for carbon nanotube composites and their potential applications as metamaterials," in 2007 
IEEE/MTT-S International Microwave Symposium, June 2007, pp. 11371140.

[37] — "Mean-field electrodynamic theory of aligned carbon nanotube composites," IEEE Transactions on Antennas and Propagation, vol. 57, no. 5, pp. 1412-1419, May 2009.

[38] S. Mikki and A. Kishk, "An efficient algorithm for the analysis and design of carbon nanotube photonic crystals," Progress In Electromagnetics Research $C$, vol. 83, pp. 83-96, 2018. 